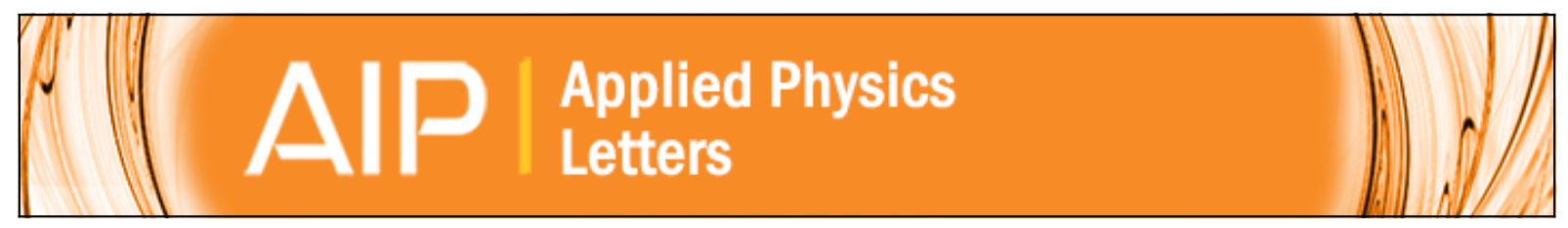

\title{
Modeling the breakdown spots in silicon dioxide films as point contacts
}

J. Suñé, E. Miranda, M. Nafría, and X. Aymerich

Citation: Applied Physics Letters 75, 959 (1999); doi: 10.1063/1.124566

View online: http://dx.doi.org/10.1063/1.124566

View Table of Contents: http://scitation.aip.org/content/aip/journal/apl/75/7?ver=pdfcov

Published by the AIP Publishing

\section{Instruments for advanced science}
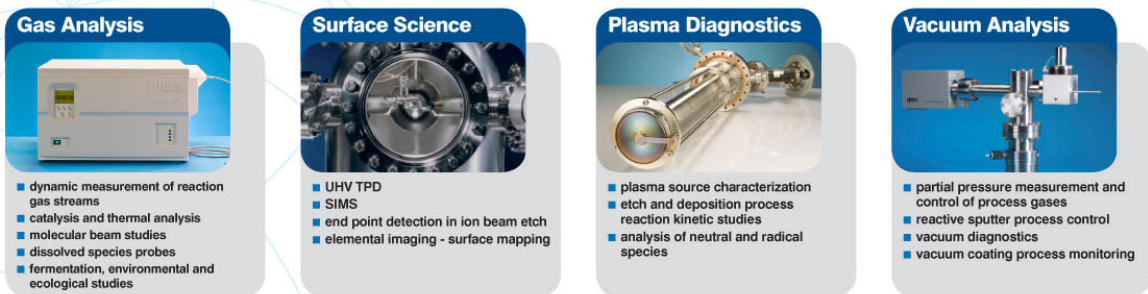

contact Hiden Analytical for further details

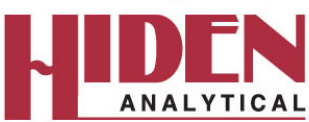

info@hideninc.com www.HidenAnalytical.com CLICK to view our product catalogue 


\title{
Modeling the breakdown spots in silicon dioxide films as point contacts
}

\author{
J. Suñé, ${ }^{\text {a) }}$ E. Miranda, ${ }^{\text {b) }}$ M. Nafría, and X. Aymerich \\ Departament d'Enginyeria Electrònica, Universitat Autònoma de Barcelona, 08193-Bellaterra, Spain
}

(Received 20 May 1999; accepted for publication 20 June 1999)

\begin{abstract}
Experiments and simulations are combined to demonstrate that the hard dielectric breakdown of thin $\mathrm{SiO}_{2}$ films in polycrystaline silicon/oxide/semiconductor structures leads to the formation of conduction paths with atomic-size dimensions which behave as point contacts between the silicon electrodes. Depending on the area of the breakdown spots, the conduction properties of the breakdown paths are shown to be those of a classical Sharvin point contact or of a quantum point contact. (C) 1999 American Institute of Physics. [S0003-6951(99)02433-X]
\end{abstract}

The hard dielectric breakdown (BD) of $\mathrm{SiO}_{2}$ films in metal-oxide-semiconductor (MOS) structures is known to be related to the generation of defects. ${ }^{1-4}$ However, little is known about the actual BD mechanism except that it is an extremely local phenomenon. Models of the BD statistics provide an estimation for the BD spot area of $10^{-12}-10^{-14}$ $\mathrm{cm}^{2}{ }^{3,4}$ If the series impedance of the sample and/or that of the measurement setup are large, thermal effects can be avoided or at least kept to a minimum. ${ }^{5,6}$ In this case, the BD spot can indeed have lateral dimensions of the order of 1-10 $\mathrm{nm}$ as the BD statistics predict. Thus, being conduction channels with both length (the oxide thickness) and lateral dimensions in the nanometer range, it is likely that the BD paths behave as ballistic point contacts. In this letter, we confirm this assumption showing that the BD paths behave as classical Sharvin point contacts (SPC) ${ }^{7,8}$ or quantum point contacts $(\mathrm{QPC})^{9-13}$ depending on the area of the BD spot. The idea of relating $\mathrm{BD}$ to the formation of quantum wires has also been independently suggested by Ting in a recent letter. ${ }^{14} \mathrm{Al}-$ though our model is conceptually very similar, the approach is completely different and we have found that a single wire of reasonable dimensions can explain the post BD $I(V)$. On the contrary, Ting's simulations correspond to a matrix of quantum wires with total area of about $10 \%$ of the capacitor area. Clearly, such a matrix of wires is a much less likely picture for oxide $\mathrm{BD}$.

Polycrystaline silicon gate MOS capacitors with an oxide thickness ranging from 2.1 to $3.8 \mathrm{~nm}$ and areas ranging from $10^{-3}$ to $10^{-5} \mathrm{~cm}^{2}$ have been fabricated onto $n$-type (100) silicon with a doping of $10^{15} \mathrm{~cm}^{-3}$. The postbreakdown conduction properties of these ultrathin oxides are analyzed and compared with those of thicker oxides [13.5 $\mathrm{nm} \mathrm{SiO} \mathrm{Si}_{2}$ films onto $n$-type (100) silicon with $N_{D}$ $\left.=10^{18} \mathrm{~cm}^{-3}\right] \cdot{ }^{6}$ In this work, we are only concerned with hard breakdown and not with soft breakdown. ${ }^{15}$ After the detection of a BD event, the current-voltage characteristics $I(V)$, is measured, and the static conductance-voltage characteristics, $G(V)$, is obtained by numerical derivation. To explain the obtained results, we propose that each BD event opens a conduction path through the oxide which behaves as

\footnotetext{
${ }^{a)}$ Electronic mail: Jordi.Sune@uab.es

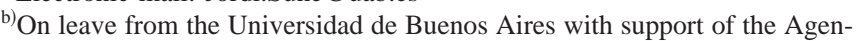
cia Española de Cooperación Internacional.
}

a point contact between the silicon electrodes. This means that no oxide barrier remains at the local position of the BD spot, but it does not necessarily require a local penetration of the electrode material into the oxide because resonant tunneling through strategically located defects can provide a transparent one-dimensional path as well. ${ }^{16} \mathrm{~A}$ very narrow conducting wire connecting two electrodes is a SPC if the electron transport is ballistic. If, in addition, the lateral dimensions of the wire are comparable to the electrons' wavelength, only a finite number of transverse modes are allowed, and it behaves like a QPC. ${ }^{9,10}$ In the latter case, the conductance takes values which are integer and half-integer multiples of the conductance quantum $G_{0}=2 e^{2} / h$, at low and high bias, respectively, $e$ and $h$ being the electron charge and $h$ the Planck's constant. ${ }^{11,12}$ To deal with point contacts between 3D silicon electrodes, we have developed an original compact model both for SPCs and QPCs. We have considered: (1) an adiabatic approach to the electron transport, ${ }^{17}$ (2) a potential profile which is adequate for semiconductor electrodes, and (3) the electron injection from the different bands of the cathode to those of the anode.

The applied voltage is considered to drop in the cathode $\left(\varphi_{S}^{C}\right)$, the anode $\left(\varphi_{S}^{A}\right)$, and in a distance $t_{\text {int }} / 2$ across each interface $\left(V_{\text {int }}\right)$ as it is schematically shown in Fig. 1. In agreement with Pascual et al. ${ }^{12}$ it is assumed that no voltage drops in the oxide path. Following the usual approach for metals, ${ }^{12}$ a fraction $\beta V_{\text {int }}$ is considered to drop at the cathode interface and the remainder $(1-\beta) V_{\text {int }}$, across the anode interface. The voltage distribution across the whole structure is calculated as in MOS structure where the nominal oxide thickness is substituted by $t_{\text {int }}$ (see Fig. 1):

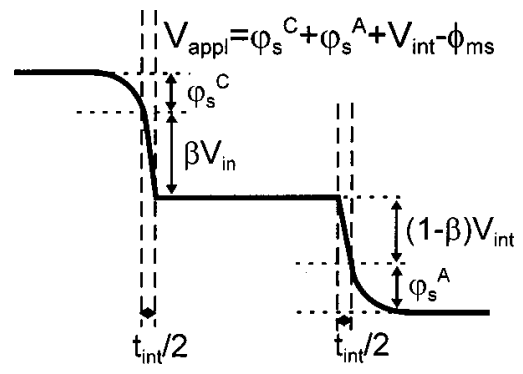

FIG. 1. Schematic representation of the potential profile at a BD spot. The main difference with the usual models used for metal point contacts (see Ref. 12) are the voltage drops in the semiconductor electrodes, $\varphi_{S}^{C}$ and $\varphi_{S}^{A}$. 


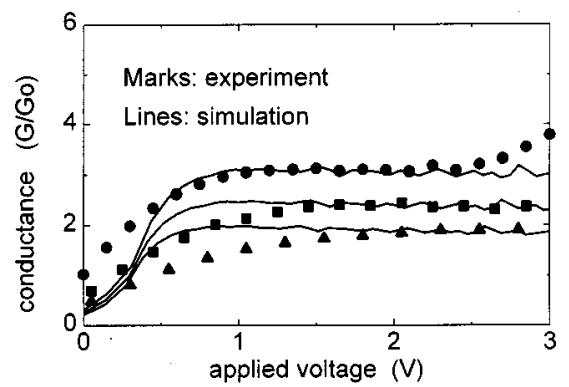

FIG. 2. Marks: experimental conductance-voltage characteristics for single breakdown spots opened in three oxides of different thickness: circles, squares, and triangles correspond to $2.1,3$, and $3.8 \mathrm{~nm}$ thick oxides, respectively. Lines: simulation results corresponding to classical Sharvin point contacts with different values of area and $t_{\text {int }}\left(\mathrm{t}_{\mathrm{int}}=2.8,2.5\right.$, and $2.2 \mathrm{~nm}$; $A=8.5 \times 10^{-13} \mathrm{~cm}^{2}, 6.5 \times 10^{-13} \mathrm{~cm}^{2}$, and $5 \times 10 \mathrm{~cm}^{2}$, respectively).

$$
\begin{aligned}
V_{\mathrm{APPL}}= & -\Phi_{\mathrm{MS}}+\varphi_{S}^{C}\left(N_{C}, V_{\mathrm{int}}, t_{\mathrm{int}}\right)+\left|\varphi_{S}^{A}\left(N_{A}, V_{\mathrm{int}}, t_{\mathrm{int}}\right)\right| \\
& +V_{\mathrm{int}},
\end{aligned}
$$

where $V_{\text {APPL }}$ is the applied voltage, $N_{C}$ and $N_{A}$ the cathode and anode dopings, and $\Phi_{\mathrm{MS}}$ the work function difference.

Assuming an adiabatic model of transport, i.e., considering that the conducting modes have transmission coefficient equal to 1 , we calculate the current components between the conduction bands, between the valence bands, and from the cathode's valence band to the anode's conduction band. In SPCs, there is not quantization of transverse energy $E_{\perp}$, and the current is given by

$I=\frac{e m^{*} S}{2 \pi^{2} \hbar^{3}} \int d E_{z} \int\left[f\left(E_{z}, E_{\perp}, E_{F}^{C}\right)-f\left(E_{z}, E_{\perp}, E_{F}^{A}\right)\right] d E_{\perp}$,

where $\hbar$ is the Planck's constant, $S$ the spot area, $m^{*}$ the effective electron mass, $e$ the electron charge, $E_{z}$ the longitudinal energy, $E_{F}$ the Fermi level, and $f$ the Fermi-Dirac distribution. The indices $C$ and $A$ indicate cathode and anode, respectively. The energy integration limits depend on the considered cathode and anode bands. The fitting parameters of our SPC model [Eqs. (1) and (2)] are $S$ and $t_{\text {int }}$. In QPCs, the number of transversal modes in the channel is finite, and the current is given by

$$
I=\sum_{n=1}^{N} g(n) \frac{e}{\pi \hbar} \int\left[f\left(E_{z}, E_{F}^{C}\right)-f\left(E_{z}, E_{F}^{A}\right)\right] d E_{z},
$$

where $N$ is the total number of modes, and $g(n)$ the corresponding degeneracy. The integration limits depend on the bands considered in the electrodes and also on the corresponding mode transversal energy, $E_{\perp n}$. In this letter, we have only considered single-mode conduction channels. Thus, the QPC model [Eqs. (1) and (3)] only contains two fitting parameters: $t_{\text {int }}$ and $E_{\perp n}$. In both point contact models, the total current is calculated as a sum of the contributions of the conduction and valence bands of both electrodes.

Figure 2 compares the $G(V)$ of BD paths opened in ultrathin oxides of various thickness $(2.1,3$, and $3.8 \mathrm{~nm}$ ) with the simulation of SPCs with different values of $t_{\text {int }}$ and different areas: $t_{\text {int }}=2.8,2.5,2.2 \mathrm{~nm}$ and $A=8.5 \times 10^{-13}$, $6.5 \times 10^{-13}, 5 \times 10^{-13} \mathrm{~cm}^{2}$, respectively. Notice that the spots' area are within the range predicted by $\mathrm{BD}$ statistical

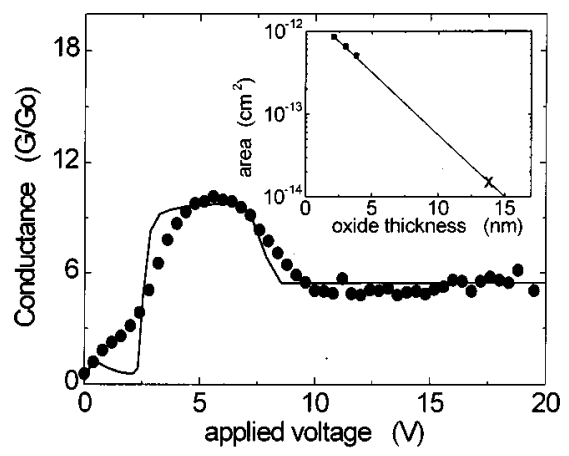

FIG. 3. Experimental conductance-voltage characteristics (marks) after the occurrence of two nearly identical breakdown events in a $n^{+}$-poly-Si/SiO $2 /$ Sistructure $\left(13.5 \mathrm{~nm}\right.$ oxide) onto a $10^{18} \mathrm{~cm}^{-3} n$-type silicon substrate. The lines correspond to the simulation of a quantum point contact with $t_{\text {int }}=3 \mathrm{~nm}$ and one single transverse mode in the channel (BD path) located at $2.5 \mathrm{eV}$ below the conduction band at flatband. Inset: Area of the $\mathrm{BD}$ spot required to fit the data of Fig. 2 as a function of the oxide thickness. Extrapolation to a $13.5 \mathrm{~nm}$ oxide gives $S=1.7 \times 10^{-14} \mathrm{~cm}^{2}$.

models ${ }^{3,4}$ and increase with decreasing thickness. In all the cases, a conductance plateau is found. At low bias the conductance is lower due to the major role of voltage drops in the electrodes. The main qualitative and quantitative features of the experimental temperature dependence of the BD current have also been shown to be captured by the SPC model. ${ }^{18}$ Attempts to model the behavior of the BD paths in these ultrathin oxides with a QPC model always yield worse results. On the contrary, the $\mathrm{BD}$ data corresponding to slightly thicker oxides $(13.5 \mathrm{~nm})^{6}$ is better understood when quantum effects are considered. Figure 3 compares the $G(V)$ of a broken down $13.5 \mathrm{~nm}$ oxide with the simulation of a QPC. Two BD events of nearly identical magnitude had been registered (see Fig. 11 of Ref. 6) so that the simulation corresponds to two QPCs conducting in parallel with $t_{\text {int }}=3 \mathrm{~nm}$ and the mode located at $E_{\perp n}=-2.5 \mathrm{eV}$ below the silicon conduction band at flatband. The degeneracy $g(n)$ has been considered to be 6 because this gives the better fit of the $I(V)$. Recall, however, that the conduction band of Si has 6 equivalent valleys. Two conductance plateaus are obtained, one at about $10 G_{0}$ and the other at $6 G_{0}$. In both cases the current is controlled by injection from the cathode's valence band towards the anode's conduction band. The transition between both plateaus occurs at the crossing of the bottom of the transversal subband and the anode's conduction band. After the crossing, the conductance of $6 G_{0}$ is easily understood: we have two spots and sixfold degeneracy so that the contribution of each single transverse mode is of about $0.5 G_{0}$, which is the value expected for the nonlinear injection regime when $\beta=1 / 2 .^{12}$ Before the crossing, the conductance does not reach $12 G_{0}$, i.e., $G_{0}$ per conducting mode, because of the voltage drops in the silicon electrodes.

Why the BD spots in ultrathin oxides behave as SPC while a QPC model has been invoked for a thicker film? This probably has to do with the area of the BD spots. The inset of Fig. 3 shows the values of $S$ used to fit the data of Fig. 2 as a function of the oxide thickness. Rough extrapolation to a $13.5 \mathrm{~nm}$ oxide gives an area more than one order of magnitude smaller, and this justifies the need of a quantum model because the lateral dimensions of the BD spot are of the order of the electrons' wavelength. 
In conclusion, the combined use of experiments and simulations has shown that the BD paths in thin $\mathrm{SiO}_{2}$ films behave as point contacts. The area of the BD spot, which has been found to be in agreement with previous estimations based on BD statistical models, determines whether the BD path behaves as a classical or quantum point contact. The presented model of point contacts between 3D semiconductors is also original and reveals that the behavior is very similar to those of metal contacts because of the relevant role of the cathode's valence band. The obtained results not only improve our understanding of the BD physics but also open a path for the use of intentionally induced $\mathrm{BD}$ paths to fabricate nanoelectronic devices based on point contacts working in the $1 \mathrm{~V}$ range.

This work was supported by the Comisión Interministerial de Ciencia y Tecnología under Project No. PB96-1162.

${ }^{1}$ D. J. DiMaria, E. Cartier, and D. Arnold, J. Appl. Phys. 73, 3367 (1993).

${ }^{2}$ K. F. Schuegraf and C. Hu, IEEE Trans. Electron Devices ED-41, 761 (1994).

${ }^{3}$ J. Suñé, I. Placencia, N. Barniol, E. Farrés, F. Martín, and X. Aymerich, Thin Solid Films 185, 347 (1990).
${ }^{4}$ R. Degraeve, G. Groseneken, R. Bellens, J. L. Ogier, M. Depas, P. J. Roussel, and H. E. Maes, IEEE Trans. Electron Devices 45, 472 (1998).

${ }^{5}$ J. C. Jackson, T. Robinson, O. Oralkan, D. J. Dumin, and G. A. Brown, Appl. Phys. Lett. 71, 3682 (1997).

${ }^{6}$ M. Nafría, J. Suñé, and X. Aymerich, J. Appl. Phys. 73, 205 (1993).

${ }^{7}$ Yu. V. Sharvin, Sov. Phys. JETP 21, 655 (1965).

${ }^{8}$ A. G. M. Jansen, A. P. van Gelder, and P. Wyder, J. Phys. C 13, 6073 (1980).

${ }^{9}$ B. J. van Wees, H. van Houten, C. W. Beenakker, J. G. Williamson, L. P. Kouwenhoven, D. Van der Marel, and C. T. Foxon, Phys. Rev. Lett. 60, 848 (1988).

${ }^{10}$ D. A. Wharam, T. Thornton, R. Newbury, M. Pepper, H. Ahmed, E. Frots, D. Hasko, D. Peacock, D. Ritchie, and G. Jones, J. Phys. C 21, L209 (1988).

${ }^{11}$ E. Castaño and G. Kircenow, Phys. Rev. B 41, 3874 (1990).

${ }^{12}$ J. I. Pascual, J. A. Torres, and J. J. Sáenz, Phys. Rev. B 55, R16029 (1997).

${ }^{13}$ N. K. Patel, J. Nicholls, L. Martín-Moreno, M. Pepper, J. Frost, D. Ritchie, and G. Jones, Phys. Rev. B 44, 13549 (1991).

${ }^{14}$ D. Z.-Y. Ting, Appl. Phys. Lett. 74, 585 (1999).

${ }^{15}$ E. Miranda, J. Suñé, R. Rodríguez, M. Nafría, and X. Aymerich, Appl. Phys. Lett. 73, 490 (1998).

${ }^{16}$ C. C. Wan, J.-L. Mozos, G. Taraschi, J. Wang, and H. Guo, Appl. Phys. Lett. 71, 419 (1997).

${ }^{17}$ H. Xu, Phys. Rev. B 47, 15630 (1993).

${ }^{18}$ J. Suñé, E. Miranda, M. Nafría, and X. Aymerich, IEEE-IEDM'98 Tech. Dig., pp. 191-194 (1998) 